\title{
ANALYTIC FUNCTIONS ON SOME RIEMANN SURFACES
}

\author{
NOBUSHIGE TODA and KIKUJI MATSUMOTO
}

1. Some years ago, Kuramochi gave in his paper [5] a very interesting theorem, which can be stated as follows.

Theorem of Kuramochi. Let $R$ be a hyperbolic Riemann surface of the class $O_{H B}\left(O_{H D}\right.$, resp. $)$. Then, for any compact subset $K$ of $R$ such that $R-K$ is connected, $R-K$ as an open Riemann surface belongs to the class $O_{A B}\left(O_{A D}\right.$, resp.).

The excellent work of Constantinescu and Cornea [2] clarified that as for the $B$ part of this theorem, the existence of a bounded minimal harmonic function, that is, the existence of a Martin boundary point with positive harmonic measure and, as for the $D$ part, the existence of an HD-minimal function are essential. Later Kusunoki and Mori [8] and Nakai [9] proved the equivalence of the existence of an $H D$-minimal function and the existence of a point with positive harmonic measure in Royden's harmonic boundary of the Riemann surface $R$. But there remains the question as to whether there exists a hyperbolic Riemann surface which has no Martin or Royden boundary point with positive harmonic measure and has yet the same property as stated in Theorem of Kuramochi.

The main purpose of this paper is to give a positive answer to the Martin part of the above question. In the sequel, we shall give an extension of the $B$ part of Theorem of Kuramochi and, using this extension, construct an open Riemann surface, as a covering surface of the complex plane, which answers the Martin part of the question in the positive.

2. First we shall be concerned with the boundary behaviour of bounded analytic functions on a Riemann surface of the class $O_{A B}^{\circ}$. The class $O_{A B}^{>}$was introduced and investigated first by Kuroda in his paper [7]. It is a class of

Received March 4, 1963. 
open Riemann surfaces, any subregion of which belongs to the class $S O_{A B}$, that is, admits no non-constant single-valued bounded analytic function with real part vanishing continuously on its relative boundary. Here a subregion of a Riemann surface $R$ means a subdomain of $R$ with smooth relative boundary clustering nowhere in $R$. It is known that the following inclusions hold.

\begin{tabular}{|c|c|c|c|}
\hline$O_{H B}$ & $\subsetneq$ & $O_{A B}^{\circ}$ & $\risingdotseq$ \\
\hline Hh & & & \\
\hline$O_{H D}$ & & $\subsetneq$ & \\
\hline
\end{tabular}

On the other hand, no inclusion holds between $O_{H D}$ and $O_{A B}^{\circ}$ or $O_{A B}$.

Let $R$ be an arbitrary open Riemann surface, and let $\left\{G_{n}\right\}_{n=1,2, \ldots}$ denote a sequence of non-compact subregions of $R$ with compact relative boundary $\partial G_{n}$ such that $G_{n} \supset G_{n+1} \cup \partial G_{n+1}$ for each $n$ and $\cap_{n=1}^{\infty} G_{n}=\emptyset$. We classify such sequences with the following equivalence relation: Two sequences $\left\{G_{n}^{1}\right\}_{n=1,2}, \ldots$ and $\left\{G_{n}^{2}\right\}_{n=1,2, \ldots}$ are equivalent if and only if, for any $m$, there is an $n$ such that $G_{n}^{1} \supset G_{m}^{2}$, and vice versa. Each of these equivalence classes is a boundary component of $R$ in Kerékjártó-Stoïlow's sense, and we consider it as an ideal boundary point of $R$. A neighborhood of this ideal boundary point $P$ means the union of $P$ and an open set of $R$ containing $G_{m} \in\left\{G_{n}\right\}_{n=1,2, \ldots}$ for some $m$, where $\left\{G_{n}\right\}_{n=1,2, \ldots}$ is a representative member of $P$.

Now let $P$ be an ideal boundary point of $R$ and let $D$ be a subdomain such that $P$ can be approached by a sequence of points in $D$. For a single-valued meromorphic function $w=f(p)$ of $D$, we shall consider the cluster set $C_{D}(f, P)$ of $f(p)$ at $P$. The cluster set $C_{D}(f, P)$ is the set of values $\alpha$ such that there exists a sequence of points $\left\{p_{n}\right\}$ of $D$ tending to $P$ and satisfying that

$$
\lim _{n \rightarrow \infty} f\left(p_{n}\right)=\alpha \text {. }
$$

Let $R$ be an open Riemann surface of the class $O_{A B}^{\circ}$ and let $K$ be a compact subset such that $R-K$ is connected. Let $w=f(p)$ be a non-constant single-valued meromorphic function on $R-K$. We consider a disc $(c):\left|w-w_{0}\right|$ $<\rho^{1 i}$ such that, for some relatively compact subregion $R_{0} \supset K$ of $R$ with smooth boundary $\partial R_{0}, f(p)$ takes on $\partial R_{0}$ no value in the closure of $(c)$. Then each connected component $\Delta$ of the inverse image $f^{-1}((c))$ on $R-\bar{R}_{0}$, if

1) In case $w_{0}=\infty$, we consider as $(c)$ a domain $|w|>\rho$. 
exist, belongs to the class $S O_{A B}$ and it follows from Theorem 5 in Kuroda [7] that the set $(c)-f(\Delta)$ is totally disconnected. Therefore the following theorem is an immediate consequence of a localization of so-called Stoilow's principle on Iversen's property ${ }^{2)}$.

THEOREM 1. Let $R$ be an open Riemann surface belonging to the class $O_{A B}^{\circ}$. Then, for any single-valued meromorphic function $w=f(p)$ defined on $R-K$, where $K$ is a compact subset such that $R-K$ is connected, the cluster set $C_{R-K}(f, P)$ is total or reduces to one point at each ideal boundary point $P$ of $R$.

As a corollary of this theorem, we have

THEOREM $2^{31}$. Let $R$ be an open Riemann surface belonging to the class $O_{A B}^{\circ}$, let $K$ be a compact subset such that $R-K$ is connected and let $w=f(p)$ be a single-valued meromorphic function on $R-K$ which is bounded or has a Riemannian image of $R-K$ over the $w$-sphere with finite spherical area. Then $f(p)$ has a limit at each ideal boundary point of $R$.

Proof. If $f(p)$ is bounded, then $C_{R-K}(f, P)$ is not total. Hence by Theorem $1 C_{R-K}(f, P)$ reduces to one point, so that our assertion follows.

Next suppose that the Riemannian image $\Phi$ of $R-K$ by $f(p)$ has a finite spherical area. If $C_{R-K}(f, P)$ is total, then we see by Theorem 5 in [7] that $\Phi$ covers the whole $w$-plane infinitely often except for at most a union of a countable number of closed sets of the class $W$ in the sense of Kametani [4] ( = the class $N_{\mathfrak{B}}$ in the sense of Ahlfors and Beurling [1]). Hence the spherical area of $\Phi$ must be infinite. Contradiction. Therefore $C_{R-K}(f, P)$ is also one point in the second case.

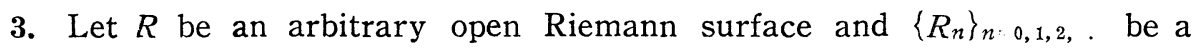
normal exhaustion of $R$ such that $R-\bar{R}_{0}$ is connected. For an ideal boundary point $P$ of $R$, this exhaustion determines a representative member $\left\{G_{n}\right\}$ of $P$ such that every $G_{n}$ is a connected component of $R-\bar{R}_{n}$. We consider the harmonic function $\omega_{n, m}(p)\left(0<\omega_{n, m}(p)<1\right)$ in $R_{m}-\bar{G}_{n}-\bar{R}_{0}(m \geqq n)$ with

2) Cf. K. Noshiro [10], Chapt. IV, $\leqslant 2$.

3) See Kuramochi [6]. There he proved the same result in the case where $f(z)$ is bounded. 
boundary values such that

$$
\omega_{n, m}(p)= \begin{cases}0 & \text { on }\left(\partial R_{m}-G_{n}\right) \cup \partial R_{0} \\ 1 & \text { on } \partial G_{n} .\end{cases}
$$

$\omega_{n, m}(\not)$ increases as $m \rightarrow \infty$ so that $\omega_{n, m}(\not)$ tends to a harmonic function $\omega_{n}(p)$. Now let $n$ tend to infinity. Then $\omega_{n}(p)$ decreases and tends to a nonnegative harmonic function $\omega_{P}(p)$ defined on $R-\bar{R}_{0} . \quad \omega_{P}(p) \equiv 0$ or $\omega_{P}(p)>0$ in $R-\bar{R}_{0}$, and we say that the harmonic measure of an ideal boundary point $P$ is zero or positive according as the first or the second case occurs, respectively. Of course this property of an ideal boundary point $P$ does not depend on the choice of the exhaustion $\left\{R_{n}\right\}$ of $R$.

Now we prove an extension of Theorem of Kuramochi stated in $\S 1$.

TheOREM 3. Let $R$ be a Riemann surface of the class $O_{A B}^{\circ}$ which has at least one ideal boundary point with positive harmonic measure. Then, for any compact subset $K$ of $R$ such that $R-K$ is connected, $R-K$ belongs to the class $O_{A B}$.

Proof. We denote by $P$ the ideal boundary point with positive harmonic measure. Let $f(p)$ be a single-valued bounded analytic function on $R-K$ and let $\left\{R_{n}\right\}_{n=0,1,2, \ldots}$ be a normal exhaustion of $R$. Then $f(p)$ has a limit at $P$ by Theorem 2. We may suppose that $|f(p)|<1$ and this limit is equal to 0 and that $R_{0} \supset K$ and $R-\bar{R}_{0}$ is connected.

We shall use the same notations for our exhaustion $\left\{R_{n}\right\}_{n=0,1,2, \ldots}$ as used in the above to define the harmonic measure of an ideal boundary point. By our assumption, the function - $\log |f(p)|$ is positive and superharmonic in $R-\bar{R}_{0}$, and, for any positive number $M$, there is an integer $n_{0}$ such that

$$
-\log |f(p)|>M \text { on } G_{n} \cup \partial G_{n} \text { for evey } n \geqq n_{0} .
$$

Therefore we have by the maximum principle that

$$
0<\omega_{n, m}(p)<\frac{1}{M}(-\log |f(p)|) \text { in } R_{m}-\bar{G}_{n}-\bar{R}_{0}
$$

for every $m(\geqq n)$. We make first $m$ and next $n$ tend to infinity so that

$$
0 \leqq \omega_{P}(p)<\frac{1}{M}(-\log |f(p)|) \text { in } R-\bar{R}_{0}
$$


Since we can take $M$ arbitrarily large and since $\omega_{P}(p)>0$ in $R-\bar{R}_{0}$, this implies that $-\log |f(p)| \equiv+\infty$, that is, $f(p)=0$. Hence we can conclude that any single-valued bounded analytic function on $R-K$ is constant. Our theorem is thus established.

4. For the proof of Theorem 3, we do not need the existence of a Martin boundary point with positive harmonic measure. In fact we can give an example of a Riemann surface which has no such a point and yet satisfies the condition of the theorem.

Construction of the example. Let $E$ be a Cantor set on the closed interval $I_{0}:[-1 / 2,1 / 2]$ with constant successive ratios $\xi_{n}, 0<\xi_{n}=2 l<1 / 3$. Then $E$ is of logarithmic positive capacity, because $E$ is of logarithmic capacity zero if and only if

$$
\sum_{n=1}^{\infty} \frac{\log \xi_{n}^{-1}}{2^{n}}=+\infty
$$

Defining the Cantor set $E$, we repeat successively to exclude an open segment from the middle of another segment and there remain $2^{n}$ segments of equal length $l^{n}$ after, beginning with the interval $I_{0}$, we repeat $n$ times. We denote these segments by $I_{n, k}\left(n=1,2, \ldots ; k=1,2, \ldots, 2^{n}\right)$. Now we consider the complementary domain $F$ of $E$ on the real axis with respect to the extended $w$-plane, and denote by $A_{n, k}\left(n=1,2, \ldots ; k=1,2, \ldots, 2^{n}\right)$ the following ring domains on $F$.

$$
A_{n, k}=\left\{l^{n}<\left|w-w_{n, k}\right|<\frac{1}{2} l^{n-1}(1-l)\right\}
$$

where $w_{n, k}$ is the middle point of $I_{n, k}$. These ring domains $A_{n, k}$ have the same harmonic modulus $\mu=(1 / l-1) / 2$ greater than $5 / 2$, and for each $n \geqq 1$, all of $A_{n+1, k}\left(k=1,2, \ldots, 2^{n+1}\right)$ together separate $E$ from all of $A_{n, k}(k=1,2$, $\left.\ldots, 2^{n}\right)$. We suppose that each $A_{n, k}\left(n=1,2 \ldots ; k=1,2, \ldots, 2^{n}\right)$ encloses $A_{n+1,2 k}$ and $A_{n+1,2 k+1}$ and denote by $\Delta_{n, k}$ the doubly-connected domain bounded by the inner boundary of $A_{n, k}$ and the outer boundaries of $A_{n+1,2 k}$ and $A_{n+1,2 k+1}$. Fix a slit $S_{n, k}$ arbitrarily in $\Delta_{n, k}$.

We shall construct a Riemann surface with only one ideal boundary component as a covering surface of $F$ in the way of Constantinescu and Cornea [3]. Let $F_{0}$ be a replica of $F$ with slits $S_{n, k}$ for all $n$ and $k(n=1,2, \ldots$; 
$\left.k=1,2, \ldots, 2^{n}\right)$ and, for each integer $m \geqq 1$, let $F_{m}$ be a replica of $F$ with slits $S_{n, k}$ only for $n$ satisfying that

$$
n=2^{m-1}(2 i+1) \quad(i=0,1,2, \ldots) .
$$

Then each slit $S_{n, k}$ appears precisely two times, once on $F_{0}$ and once on some $F_{m}(m \geqq 1)$. Connect all $F_{m}$ with $F_{0}$ crosswise across $S_{n, k}\left(n=2^{m-1}(2 i+1)\right.$ $\left.(i=0,1,2, \ldots) ; k=1,2, \ldots, 2^{n}\right)$. Then it is easily seen that the resulting surface $\widetilde{F}$ has only one ideal boundary component. Since $\widetilde{F}$ has as its projection the domain $F$ in the $w$-plane whose complement $E$ is of logarithmic positive capacity, $\widetilde{F}$ is hyperbolic and hence its only one ideal boundary component has a positive harmonic measure.

To show that $\widetilde{F}$ is a desired Riemann surface. It is enough to prove that

(i) $\widetilde{F} \in O_{A B}^{\circ}$,

(ii) $\widetilde{F}$ admits no bounded minimal harmonic function.

Proof of (i). Denoting by $A_{n, k}^{(m)}$ the ring domain on $F_{m}$ corresponding to $A_{n, k}$ on $F$, we consider on $F_{0}$ all $A_{n, m}^{(0)}\left(n=1,2, \ldots ; k=1,2, \ldots, 2^{n}\right)$ and on $F_{m}(m=1,2, \ldots) A_{n, k}^{(m)}$ only for $n$ and $k$ such that

$$
n \geqq 2^{m-1}+1 \quad \text { and } \quad k=1,2, \ldots, 2^{n} \text {. }
$$

Then these $A_{n, k}^{(m)}\left(m=0,1,2, \ldots ; n \geqq 1\right.$ if $m=0$ and $n \geqq 2^{m-1}+1$ otherwise ; $\left.k=1,2, \ldots, 2^{n}\right)$ have the same harmonic modulus $\mu=(1 / l-1) / 2$ greater than $5 / 2$, and, for each $n \geqq 1$, all of $A_{n+1, k}^{(m)}$ (all $m$ satisfying $n+1 \geqq 2^{m-1}+1$; $k=1,2, \ldots, 2^{n+1}$ ) together separate the ideal boundary of $\widetilde{F}$ from all of $A_{n, k}^{(m)}$ (all $m$ satisfying $n \geqq 2^{m-1}+1$ if $n \geqq 2$ and $m=0$ if $n=1 ; k=1,2, \ldots, 2^{n}$ ).

Here we use the following criterion due to Kuroda [7].

If a Riemann surface $R$ admits a sequence of ring domains $B_{n, k}(n=1,2$, $\ldots ; k=1,2, \ldots, \nu(n))$ such that, for each $n$, all of $B_{n+1, k}(k=1,2, \ldots$, $\nu(n+1))$ together separate the ideal boundary of $R$ from all of $B_{n, k}(k=1,2$, $\ldots, \nu(n))$ and

$$
\left.\lim _{N \rightarrow \infty} \sup _{N=1}^{N} \log \mu_{n}-\log \nu(N)\right\}=+\infty,
$$

then $R$ belongs to the class $O_{A B}^{\circ}$. Here $\mu_{n}$ denotes the minimum harmonic modulus of ring domains $B_{n, k}(k=1,2, \ldots, \nu(n))$. 
In our case, $\mu_{n}=\mu>5 / 2$ and $\nu(n) \leqq 2^{n} n$. Hence

$$
\lim \sup _{N \rightarrow \infty}\left\{\sum_{n=1}^{N} \log \mu_{n}-\log \nu(N)\right\} \geqq \lim _{N \leftarrow \infty}\left\{N \log \frac{5}{2}-\log \left(2^{N} N\right)\right\}=+\infty,
$$

so that $\widetilde{F}$ belongs to the class $O_{A B}^{\circ}$.

Proof of (ii). Constantinescu and Cornea proved in their paper [2] that if a Riemann surface $R$ admits a bounded minimal harmonic function, then any non-constant single-valued analytic function on $R$ takes every value infinitely often with possible exception of logarithmic capacity zero. The projection of $\hat{F}$ is non-constant, single-valued and analytic but takes no value of $E$ of logarithmic positive capacity. Therefore it follows from Constantinescu and Cornea's result that $\widetilde{F}$ admits no bounded minimal harmonic function.

\section{REFERENCES}

[1] L. V. Ahlfors and A. Beurling: Conformal invariants and function-theoretic nullsets, Acta Math., 83 (1950), 101-129.

[2] C. Constantinescu and A. Cornea: Über den idealen Rand und einige seiner Anwendungen bei Klassifikation der Riemannschen Flächen, Nagoya Math. J., 13 (1958), 166-233.

[ 3 ] C. Constantinescu and A. Cornea: Über einige Probleme von M. Heins, Rev. Math. pur. appl., 4 (1959), 277-281.

[4] S. Kametani: On Hausdorff's measures and generalized capacities with some of their applications to the theory of functions, Jap. J. Math., 19 (1945-48), 217-257.

[5] Z. Kuramochi: On the behaviour of analytic functions on abstract Riemann surfaces, Osaka Math. J., 7 (1955), 109-127.

[6] Z. Kuramochi: Representation of Riemann surfaces, Osaka Math. J., 11 (1959), 71-82.

[7] T. Kuroda: On analytic functions on some Riemann surfaces, Nagoya Math. J., 10 (1956), 27-50.

[8] Y. Kusunoki and S. Mori: On the harmonic boundary of an open Riemann surface, II, Mem. Coll. Sci. Univ. Kyoto, 33 (1960), 209-223.

[9] M. Nakai: A measure on the harmonic boundary of a Riemann surface, Nagoya Math. J., 17 (1960), 181-218.

[10] K. Noshiro: Cluster Sets, Berlin (1960).

\section{Mathematical Institute}

Nagoya University 